PROCEEDINGS OF THE

AMERICAN MATHEMATICAL SOCIETY

Volume 117, Number 1, January 1993

\title{
NEAR-RINGS OF INVARIANTS. II
}

\author{
C. J. MAXSON
}

(Communicated by Maurice Auslander)

\begin{abstract}
Let $E$ be the equivalence relation determined by the orbits of a group $G$ acting on a set $X$ and let $K$ be a group. Using a "sandwich" function $\varphi: X / E \rightarrow K, M(X, G, K, \varphi)=\{f: X \rightarrow K \mid f$ is constant on each $E$-class $\}$ is a near-ring, the near-ring of invariants determined by $(X, G, K, \varphi)$. In this paper we continue the study of the transfer of information between the structure of the near-ring of invariants and properties of the group action $(G, X)$, the group $K$, and the function $\varphi: K \rightarrow X / E$.
\end{abstract}

\section{INTRODUCTION}

Let $E$ be an equivalence relation on a set $X$ and let $(K,+)$ be a group, not necessarily abelian, with identity 0 . Let $\varphi: K \rightarrow X$ be a function and let $M:=M(X, E, K, \varphi)=\{f: X \rightarrow K \mid f$ is constant on each $E$-class in $X\}$. Under function addition, $(M,+)$ is a group. When we define a sandwich multiplication $*$ on $M$ by $f * g=f \varphi g$ (the usual composition of functions), $f, g \in M$, then $(M,+, *)$ is a (right) near-ring, a subnear-ring of the "full" sandwich near-ring studied by Fuchs [1]. In fact, if $E$ is the identity equivalence relation on $X$ then $M$ is the full sandwich near-ring $M(X, K, \varphi)$ of Fuchs. (Also, see Fuchs and Pilz [2].)

Let $X$ be a group and let $S$ be a group acting on $X$. We let $E$ be the equivalence relation determined by the orbits of the action of $S$ on $X$, take $K=X$, and take $\varphi$ to be the identity map on $X$. Then $M(X, E, K, \varphi)$ is the near-ring of invariants studied in [3].

As a further interpretation let $E$ be the conjugacy relation on a group $G$ determined by the inner automorphisms of $G$. Suppose we have a representation $\rho$ of $G$ by matrices over a field $K$. Then $M(G, E, K, \varphi)$ contains the near-ring generated by the characters of the representation $\rho$.

We next show that the structure of $M(X, E, K, \varphi)$ is related to a problem of Wielandt [6]. To this end, suppose a group $G$ acts on a set $X,(g, x) \mapsto x^{g}$, $x \in X, g \in G$. Following Wielandt, we say $\Phi$ is a $k$-relation on $X$ if $\Phi$ is a subset of $X^{k}$, the Cartesian product of $X$ with $k$ factors. The $k$-relation $\Phi$ is said to be $G$-invariant, denoted by $\Phi \in k-\operatorname{rel}(G)$, if $\Phi^{g}=\Phi$ for all $g \in G$, where $\Phi^{g}=\left\{\left(\alpha_{1}^{g}, \ldots, \alpha_{k}^{g}\right) \mid\left(\alpha_{1}, \ldots, \alpha_{k}\right) \in \Phi\right\}$.

Received by the editors May 22, 1991.

1991 Mathematics Subject Classification. Primary 16Y30, 20B99. 
Let $K$ be a group, $|K| \geq 2$, and let $M\left(X^{k}, K\right)=\left\{f: X^{k} \rightarrow K\right\}$, a group under function addition. For $g \in G, f \in M\left(X^{k}, K\right)$ we let $g$ act on $f$ by $f^{g}(x)=f\left(x^{g^{-1}}\right), x \in X^{k}$. It is straightforward to verify that $G$ acts as a group of automorphisms of $M\left(X^{k}, K\right)$. For $\Phi \subseteq X^{k}$ defined a function $f_{\Phi}: X^{k} \rightarrow K$ by $f_{\Phi}(x)=0$ if $x \in \Phi$ and by $f_{\Phi}(x)=a$ if $x \notin \Phi$ where $a$ is some nonzero element of $K$. Then $f_{\Phi}$ is in $M\left(X^{k}, K\right)$ and $f_{\Phi}^{g}=f_{\Phi s}$ for each $g \in G$. In fact, if $y \in \Phi^{g}$ then $y=x^{g}$ for some $x \in \Phi$, so $f_{\Phi}^{g}(y)=f_{\Phi}\left(\left(x^{g}\right)^{g^{-1}}\right)=f_{\Phi}(x)=0$; while if $y \notin \Phi^{g}$ then $y^{g^{-1}} \notin \Phi$, so $f_{\Phi}^{g}(y)=a \neq 0$, i.e., $f_{\Phi}^{g}=f_{\Phi g}$ as desired.

If we take $\operatorname{Fix}_{k}(G)=\left\{f \in M\left(X^{k}, K\right) \mid f^{g}=f\right.$ for all $\left.g \in G\right\}$ then $\Phi \in$ $k-\operatorname{rel}(G) \Leftrightarrow f_{\Phi} \in \operatorname{Fix}_{k}(G)$. For $\Phi \in k-\operatorname{rel}(G) \Leftrightarrow \Phi^{g}=\Phi \forall g \in G$. It follows from the above discussion that $f_{\Phi}^{g}=f_{\Phi}$. But $f_{\Phi}^{g}=f_{\Phi}$ also implies $\Phi^{g}=\Phi$, so we have $\Phi \in k-\operatorname{rel}(G)$ is equivalent to $f_{\Phi} \in \operatorname{Fix}_{k}(G)$.

For $f \in M\left(X^{k}, K\right)$ and $c \in K$ define $\Phi(f=c)=\left\{x \in X^{k} \mid f(x)=c\right\}$, which is a level surface for $f$.

Theorem I.1 [6]. Let a group $G$ act on a set $X$ and let $E$ denote the equivalence relation determined by the orbits of $G$. The following are equivalent:

(1) $f \in \operatorname{Fix}_{k}(G)$;

(2) $f \in M\left(X^{k}, E, K, \varphi\right)$;

(3) $\Phi(f=c) \in k-\operatorname{rel}(G), \forall c \in K$.

Proof. (1) $\Rightarrow(2)$. Let $f \in \operatorname{Fix}_{k}(G)$ and let $x, y$ be in the same orbit determined by $G$ acting on $X$. Then $x^{g^{-1}}=y$ for some $g \in G$ so $f(y)=$ $f\left(x^{g^{-1}}\right)=f^{g}(x)=f(x)$ since $f \in \operatorname{Fix}_{k}(G)$, hence $f \in M\left(X^{k}, E, K, \varphi\right)$.

(2) $\Rightarrow(3)$. Let $x \in \Phi(f=c)$. We show $x^{g} \in \Phi(f=c)$ for each $g \in$ $G$. Since $x$ and $x^{g}$ are in the same orbit, $f\left(x^{g}\right)=f(x)=c$ since $f \in$ $M\left(X^{k}, E, K, \varphi\right)$. Thus $x^{g} \in \Phi(f=c)$ so $(\Phi(f=c))^{g} \subseteq \Phi(f=c)$. But then $\Phi(f=c)=\left((\Phi(f=c))^{g^{-1}}\right)^{g} \subseteq(\Phi(f=c))^{g}$ gives the reverse inclusion.

(3) $\Rightarrow(1)$. Let $f: X^{k} \rightarrow K$ be such that $\Phi(f=c) \in k-\operatorname{rel}(G)$ for each $c \in K$, and let $g \in G, x \in X^{k}$. If $f(x)=b$ then $x \in \Phi(f=b)$. Therefore $x^{g^{-1}} \in \Phi(f=b)$. Thus $f(x)=b=f\left(x^{g^{-1}}\right)=f^{g}(x)$. Since $x$ is arbitrary, $f=f^{g}$, so $f \in \operatorname{Fix}_{k}(G)$.

If we refer to the elements of $M(X, E, K, \varphi)$ as invariant functions, we have shown that a level surface of an invariant function is an invariant $k$ relation and conversely. Thus problems in the study of invariant relations are equivalent to problems in $M(X, E, K, \varphi)$. It is hoped that information about the structure of this near-ring will provide information about invariant relations and ultimately information about permutation groups via the Wielandt program.

In our final result of this section we show that when considering structural properties, the near-ring of invariants, $M(X, E, K, \varphi)$ can be considered a full sandwich near-ring.

Theorem I.2. Let $E$ be an equivalence relation on a set $X$, let $K$ be a group, and let $M(X, E, K, \varphi)$ be the near-ring of invariants. Then $M(X, E, K, \varphi)$ is isomorphic to a full sandwich near-ring $M(X / E, K, \psi)$.

Proof. Let $\eta: X \rightarrow X / E$ be the canonical map. Then for $g: X / E \rightarrow K, g \eta$ 
maps $X$ into $K$. If $x, y$ are elements of $X$ in the same $E$-class then $\eta(x)=$ $\eta(y)$, so $g \eta \in M(X, E, K, \varphi)$. We also note that $\psi:=\eta \varphi$ maps $K$ into $X / E$. We thus have a function $\Gamma: M(X / E, K, \psi) \rightarrow M(X, E, K, \varphi), \Gamma(g)=$ $g \eta$. For $f, g \in M(X / E, K, \psi), \Gamma(f+g)=\Gamma(f)+\Gamma(g)$ and $\Gamma(f * g)=$ $(f * g) \eta=f \psi g \eta=f \eta \varphi g \eta=\Gamma(f) * \Gamma(g)$; thus $\Gamma$ is a near-ring homomorphism. If $g \in \operatorname{Ker} \Gamma$ then $g \eta=0$ in $M(X, E, K, \varphi)$, which means $g$ is 0 in $M(X / E, K, \psi)$. Further $\Gamma$ is surjective, for if $h \in M(X, E, K, \varphi)$, we let $X / E=\left\{E_{\alpha}\right\}$ and define $\hat{h}: X / E \rightarrow K$ by $\hat{h}\left(E_{\alpha}\right)=h(x)$ where $x$ is any element in $E_{\alpha}$. The function $\hat{h}$ is well defined in $M(X / E, K, \psi)$ and $\Gamma(\hat{h})=h$. Thus $\Gamma$ is an isomorphism as desired.

For the remainder of the paper we use $M(X / E, K, \psi)$ with $|X / E|>1$. We conclude this section by giving a brief outline of the paper. In the next section we characterize when $M:=M(X / E, K, \psi)$ has an identity, and in this case, we determine the group of units. We turn in $\S$ III to the internal structure, investigating when $M$ is a simple near-ring and when $M$ has no $(M, M)$-subgroups other than $M$ and the constant subnear-ring $M_{c}$. In the final section we turn to a more external viewpoint and characterize when $K$ is a $\nu$-primitive $M$-group, $\nu=0,1,2$.

\section{IDENTITY AND GROUP OF UNITS}

In this short section we determine when $M=M(X / E, K, \psi)$ has an identity and if this is the case we characterize the group of units of $M$.

Theorem II.1. Let $M=M(X / E, K, \psi)$. Then $M$ has an identity $\Leftrightarrow \psi: K \rightarrow$ $X / E$ is a bijection.

Proof. Suppose $\psi$ is a bijection and define $e: X / E \rightarrow K$ by $e\left(E_{\alpha}\right)=\psi^{-1}\left(E_{\alpha}\right)$, $\forall E_{\alpha} \in X / E$. For $f \in M$ and $E_{\alpha} \in X / E,(f * e) E_{\alpha}=f \psi \psi^{-1}\left(E_{\alpha}\right)=f\left(E_{\alpha}\right)$ and $(e * f)\left(E_{\alpha}\right)=e\left(\psi f\left(E_{\alpha}\right)=\psi^{-1}\left(\psi f\left(E_{\alpha}\right)\right)=f\left(E_{\alpha}\right)\right.$. Hence $e$ is an identity for $M$. Conversely, suppose $\psi$ is not a bijection. If $\psi$ is not surjective then for some $E_{\beta} \in X / E, E_{\beta} \notin \psi(K)$. Define $h \in M$ by $h\left(E_{\beta}\right)=k_{0} \in K$ and for $E_{\gamma} \neq E_{\beta}, h\left(E_{\gamma}\right)=k_{1} \neq k_{0}$. There is no function $k \in M$ such that $h * k=h$ so there are no right identities. In a similar manner, if $\psi$ is not injective, say $\psi\left(k_{1}\right)=\psi\left(k_{2}\right), k_{1} \neq k_{2}$, then if we define $g \in M$ by $g\left(E_{\alpha^{\prime}}\right)=k_{1}$ and $g\left(E_{\beta}\right)=k_{2}$ for $\beta \neq \alpha^{\prime}$ we find there are no left identities.

We now suppose $M$ has an identity, $e$, so from the above theorem, $\psi$ is a bijection. Thus for each $f \in M(X / E, K, \psi), f \psi \in M(K)=\{g: K \rightarrow K\}$. We now show that the function $\Phi: M \rightarrow M(K), f \mapsto f \psi$ is an isomorphism. Note first that $\Phi$ is a homomorphism, for if $f, g \in M$ then $\Phi(f * g)=$ $(f * g) \psi=(f \psi g) \psi=f \psi g \psi=\Phi(f) \Phi(g)$. Further, if $f \in \operatorname{Ker} \Phi$ then $f \psi=0_{K}$, so $f=f \psi \psi^{-1}=0 \psi^{-1}=0$. Hence $\Phi$ is injective. Finally, for $g \in M(K), g \psi^{-1} \in M$ and $\Phi\left(g \psi^{-1}\right)=g$.

Theorem II.2. If $M=M(X / E, K, \psi)$ has an identity then $M \cong M(K)$.

If we let $\operatorname{Un}(M)$ denote the group of units of $M$ and let Perm $(K)$ denote the bijections in $M(K)$ then we have

Corollary II.3. For $M=M(X / E, K, \psi), \operatorname{Un}(M) \cong \operatorname{Perm}(M)$.

We note that if $C(n)$ denotes the cyclic group of order $n$ and if $X$ is a set with an equivalence relation $E$ such that $|X / E|=n$, then, if we let $\psi$ be any 
bijection, $\psi: K \rightarrow X / E$, we have $\operatorname{Un}(M) \cong S_{n}$ where $S_{n}$ is the symmetric group on $n$-symbols and $M=M(X / E, C(n), \psi)$. In this case the identity for the near-ring $M$ is not the identity function.

\section{SIMPLICITY AND $(M, M)$-SIMPLICITY}

We next turn to a study of the internal structure of a near-ring $M(X / E, K, \psi)$ of invariants. We are particislarly interested in the two-sided ideals of $M$ and the $(M, M)$-subgroups of $M$, i.e., those subgroups $(B,+)$ of $(M,+)$ such that $M B \subseteq B$ and $B M \subseteq B$. To this end, the set $\mathscr{U}=\{H \unlhd K \mid H+y \subseteq$ $\left.\psi^{-1} \psi(y) \forall y \in K\right\}$ will be very useful.

We note that $\{0\} \in \mathscr{U}$, and by taking $y=0$, we see that if $H \in \mathscr{U}$ then $H \subseteq \psi^{-1} \psi(0)$. Further, the translation property, $H+y \subseteq \psi^{-1} \psi(y)$ implies that each class $\psi^{-1} \psi(y)$ is a union of cosets of $H$. In fact, $\psi^{-1} \psi(y)=$ $\bigcup_{\psi(x)=\psi(y)}(H+x)$. For if $\psi(x)=\psi(y)$ then $\psi^{-1} \psi(x)=\psi^{-1} \psi(y)$, so $H+x \subseteq$ $\psi^{-1} \psi(y)$; if $\mu \in \psi^{-1} \psi(y)$ then $\psi(u)=\psi(y)$ and $u \in H+u$.

Let $H_{1}, H_{2} \in \mathscr{U}$ and define $H_{1} \leq H_{2}$ if $H_{1} \subseteq H_{2}$. It is straightforward to show that $H_{1} \cap H_{2} \in \mathscr{U}$. Further, for each $h_{2} \in H_{2}$ and each $y \in K$, $H_{1}+\left(h_{2}+y\right) \subseteq \psi^{-1} \psi(y)$. Since $H_{2} \in \mathscr{U}$ it follows that $\psi\left(h_{2}+y\right)=\psi(y)$, so we have $H_{1}+H_{2} \in \mathscr{U}$. Thus $\mathscr{U}$ is a lattice with a unique minimal element $\{0\}$. Note also that $K \in \mathscr{U}$ if and only if $|\psi(K)|=1$, so, in general, $K \notin \mathscr{U}$. However, there is always a unique maximal element in $\mathscr{U}$. The existence of maximal elements follows from Zorn's lemma. For, if $\mathscr{C}=\left\{H_{i}\right\}$ is a chain in $\mathscr{U}$ then $\bigcup H_{i} \in \mathscr{U}$. Hence $\mathscr{U}$ has maximal elements. On the other hand, if $H^{\prime}, H^{\prime \prime}$ are maximal in $\mathscr{U}$ then, since $H^{\prime}+H^{\prime \prime} \in \mathscr{U}, H^{\prime}=H^{\prime \prime}$.

Fuchs [1] has described the ideals of $M(Y, K, \psi)$ for any set $Y$ and has given necessary and sufficient conditions for the near-ring $M(Y, K, \psi)$ to be simple. Further, in [3], with $X=K$, with $|X / E|<\infty$, and with $\psi$ equal to the canonical map $\psi: K \rightarrow K / E$, we determined the ideals of $M:=$ $M(K / E, K, \psi)$ as well as the radicals $J_{\nu}(M)$ and the quotient structures $M / J_{\nu}(M), \nu=0,1,2$.

We return to the general situation. Suppose $|K|=2$. If $\psi(K) \neq X / E$ then $\operatorname{Ann}_{M}(\psi(K))=(0: \psi(K))_{M}$ is an ideal of $M$ with $\{0\} \varsubsetneqq \operatorname{Ann}_{M}(\psi(K)) \varsubsetneqq M$. If $\psi(K)=X / E$ then we have that $\psi$ is a bijection since we do not have $|X / E|=1$. Therefore, from Theorem II.2, $M \cong M(K) \cong M\left(\mathbb{Z}_{2}\right)$, which is not simple.

Theorem III.1. If $|K|=2$ then $M(X / E, K, \psi)$ is not simple.

The next result follows from Theorem 6.1 of [1]. We also provide a short sketch of the proof.

Theorem III.2 [1, 3]. If $|K| \geq 3$ and $|X / E|<\infty$ then $M(X / E, K, \psi)$ is simple $\Leftrightarrow \psi$ is surjective and $\mathscr{U}=\{\{0\}\}$.

Proof (Sketch). If $\psi(K) \neq X / E$ then $\operatorname{Ann}_{M}(\psi(K))$ is an ideal of $M$ with $\{0\} \subsetneq \operatorname{Ann}_{M}(\psi(K)) \subsetneq M$. If $\{0\} \subsetneq H \in \mathscr{U}$ then $I=\{f \in M \mid f(X / E) \subseteq H\}$ is an ideal of $M$ with $\{0\} \subsetneq I \subsetneq M$. For the converse, let $A$ be an ideal of $M$. If $\{0\} \subsetneq A \subsetneq M$ and $A \nsubseteq M_{c}$ then as in [3], one gets $\{0\} \neq H \in U$, a contradiction. If $\{0\} \subsetneq A \subsetneq M$ and $A \supseteq M_{c}$ then, again following [3], one gets $\{0\} \neq \psi^{-1} \psi\{0\}$ and $\psi^{-1} \psi\{0\} \in \mathscr{U}$, also a contradiction. 
Now let $B$ be an $(M, M)$-subgroup of $M$. We note that for each $a \in K$ the constant function $c_{a}: X / E \rightarrow K$ is in $B$, since for each $b \in B, c_{a}=$ $c_{a} * b \in B$. Hence $M_{c}$ is contained in each $(M, M)$-subgroup of $M$. We say $M$ is $(M, M)$-simple if the only $(M, M)$-subgroups of $M$ are $M$ and $M_{c}$. We turn to the problem of characterizing those $M(X / E, K, \psi)$ that are $(M, M)$-simple.

Suppose first that $\psi: K \rightarrow X / E$ is not surjective. If $|\psi(K)| \neq 1$ then $|X / E| \geq 3$ since we know $|X| E \mid \neq 1$. Let $T=\{f \in M \mid f$ is constant on $\psi(K)\}$ and note that $T<M$ with $M_{c} \subseteq T$. Since $\psi(K) \neq X / E, T \supsetneqq M_{c}$, and since $|\psi(K)|>1$, we have that $T \neq M$. Let $E_{\alpha}, E_{\beta} \in \psi(K), t \in T$, and $f \in M$. Then $(f * t)\left(E_{\alpha}\right)=(f * t)\left(E_{\beta}\right)$, hence $f * t \in T$. On the other hand, $t * f \in M_{c}$ for each $t \in T, f \in M$, so $T$ is an $(M, M)$-group with $M_{c} \varsubsetneqq T \varsubsetneqq M$, i.e., $M$ is not $(M, M)$-simple.

If $|\psi(K)|=1$ then, for each $f, g \in M, f * g \in M_{c}$. In fact, for $E_{\alpha}, E_{\beta} \in$ $X / E,(f * g)\left(E_{\alpha}\right)=f \psi\left(g E_{\alpha}\right)=f \psi\left(g E_{\beta}\right)=(f * g)\left(E_{\beta}\right)$. From this we find that if $T<M$ with $T \supseteq M_{c}$ then $T$ is an $(M, M)$-subgroup of $M$. Let $K^{\prime}$ be a subgroup of $K,\{0\} \subsetneq K^{\prime} \varsubsetneqq K$ and let $E_{\gamma} \in X / E$. Define $A\left(E_{\gamma} ; K^{\prime}\right)=$ $\left\{f \in M \mid f\left(E_{\gamma}\right)=0\right.$ and $\left.f\left(E_{\alpha}\right) \in K^{\prime}, \alpha \neq \gamma\right\}$. Then $A\left(E_{\gamma} ; K^{\prime}\right)<M$ and so in this situation $A\left(E_{\gamma} ; K^{\prime}\right)+M_{c}$ is an $(M, M)$-subgroup of $M$. Since $|X / E|>1$ it follows that $A\left(E_{\gamma} ; K^{\prime}\right) \neq\{0\}$, hence $A\left(E_{\gamma} ; K^{\prime}\right)+M_{c} \supsetneqq M_{c}$. Since $K^{\prime} \neq K$, say $k_{0} \in K \backslash K^{\prime}$, the function $g: X / E \rightarrow K$ defined by $g\left(E_{\beta}\right)=k_{0}, \beta \neq \gamma$, and $g\left(E_{\gamma}\right)=0$ is in $M$ but not in $A\left(E_{\gamma} ; K^{\prime}\right)+M_{c}$. Hence $M$ is not $(M, M)$ simple if such a $K^{\prime}$ exists, i.e., $M$ is not $(M, M)$-simple unless $K$ is a cyclic group of prime order.

Suppose $|\psi(K)|=1$ and $|X / E| \geq 3$. We let $E_{\alpha} \in X / E$ and note that $\operatorname{Ann}\left(X / E-\left\{E_{\alpha}\right\}\right)$ is a subgroup of $M$; thus $\operatorname{Ann}\left(X / E-\left\{E_{\alpha}\right\}\right)+M_{c}$ is an $(M, M)$-subgroup of $M$ properly containing $M_{c}$. Let $\left\{E_{\alpha}, E_{\beta}, E_{\sigma}\right\} \subseteq X / E$. If $h$ is any function such that $h\left(E_{\beta}\right) \neq h\left(E_{\sigma}\right)$ then $h \notin \operatorname{Ann}\left(X / E-\left\{E_{\alpha}\right\}\right)+M_{c}$. Hence $M$ is not $(M, M)$-simple.

Before continuing the discussion of $(M, M)$-simplicity we introduce some notation. When $|X / E|=n<\infty$ we can index the classes to obtain $X / E=$ $\left\{E_{1}, E_{2}, \ldots, E_{n}\right\}$. Then each function $b \in M$ can be represented as an $n$ tuple, $b=\left(b_{1}, \ldots, b_{n}\right)$ where $b\left(E_{i}\right)=b_{i}, i=1,2, \ldots, n$. Thus $M=$ $X_{i=1}^{n} K=K \times \cdots \times K$.

We now return to $|\psi(K)|=1$. We have seen that if $|X / E|<2$ then $M$ is not $(M, M)$-simple. Suppose $|X / E|=2$, say $X / E=\left\{E_{1}, E_{2}\right\}$. If we consider $\operatorname{Ann}\left(X / E-\left\{E_{2}\right\}\right)=\operatorname{Ann}\left(E_{1}\right)$ we find Ann $E_{1}=\{0\} \times K$. Thus for each $y \in K,(0,-y) \in \operatorname{Ann}\left(E_{1}\right)$ and $(y, 0)=(0,-y)+(y, y) \in \operatorname{Ann}\left(E_{1}\right)+M_{c}$. Hence $\operatorname{Ann}\left(E_{1}\right)+M_{c}=M$. Therefore, if $T$ is an $(M, M)$-subgroup of $M$ and $b=\left(b_{1}, b_{2}\right)$ is a nonconstant function in $T$, then $\left(0, b_{2}-b_{1}\right) \in T$, $b_{2}-b_{1} \neq 0$. If $K$ is cyclic of prime order then $\{0\} \times K \subseteq T$, so, as above, $M=T$. Since we have already shown that $|\psi(K)|=1,|X| E \mid=2$, and that $M$ being $(M, M)$-simple implies $K$ must be cyclic of prime order, we have established the following result.

Theorem III.3. Let $|X / E|=2$ and $|\psi(K)|=1$. Then $M$ is $(M, M)$-simple $\Leftrightarrow K$ is cyclic of prime order.

We have also established above that when $\psi$ is not surjective and $|X / E| \geq 3$ then $M$ is not $(M, M)$-simple. 
Next, we take $\{0\} \neq H \in \mathscr{U}$ and define $S(H)=\{f \in M \mid f(X / E) \subseteq H\}$. Since $H \in \mathscr{U}$, it is straightforward to verify that $S(H)$ is an ideal of $M$, consequently $S(H)+M_{c}$ is an $(M, M)$-subgroup. In fact, for $f \in S(H)$, $c_{a} \in M_{c}$, and $g \in M, g *\left(f+c_{a}\right)-g * c_{a} \in S(H)$, so $g *\left(f+c_{a}\right) \in S(H)+M_{c}$. Since $\left(S(H)+M_{c}\right) M \subseteq S(H)+M_{c}$, we have indeed that $S(H)+M_{c}$ is an $(M, M)$-subgroup of $M$. If $H \neq K$, say $k_{0} \in K-H$, then the function $g: X / E \rightarrow K$ defined by $g\left(E_{\alpha}\right)=0, g\left(E_{\beta}\right)=k_{0}$ for $\alpha \neq \beta$, is in $M$ but not in $S(H)+M_{c}$, so $M$ is not $(M, M)$-simple. If $H=K$ then $K+0 \subseteq \psi^{-1} \psi(0)$ implies $|\psi(K)|=1$. When $|X / E| \geq 3$ we know $M$ is not $(M, M)$-simple. We have established part of the following characterization result.

Theorem III.4. If $3 \leq|X / E|=n<\infty$ then $M=M(X / E, K, \psi)$ is $(M, M)$ simple $\Leftrightarrow \psi$ is surjective and $\mathscr{U}=\{\{0\}\}$.

Before showing that the conditions are sufficient we single out a lemma.

Lemma III.5. Let $|X| E \mid=n<\infty$ and let $B$ be an $(M, M)$-subgroup of $M$. If $\psi: K \rightarrow X / E$ is surjective and for some $i, i \in\{1,2, \ldots, n\}, A_{i}:=$ $\operatorname{Ann}\left(\bigcup_{j \neq i} E_{j}\right) \subseteq B$, then $B=M$.

Proof. Without loss of generality we take $i=1$. Thus, $A_{1}=K \times\{0\} \times \cdots \times$ $\{0\} \subseteq B$. Since $\psi$ is surjective, $\forall E_{i} \in X / E, \exists k_{i} \in K$ such that $\psi\left(k_{i}\right)=E_{i}$, $i=1,2, \ldots, n$. Let $h: X / E \rightarrow K$ be defined by $h\left(E_{i}\right)=k_{1}$ and $h\left(E_{j}\right)=k_{2}$ for $i \neq j$. Then for $x \in K,(x, 0, \ldots, 0) \in B$ and $(x, 0, \ldots, 0) * h=e_{i}^{x}$ where $e_{i}^{x}\left(E_{i}\right)=x$ and $e_{i}^{x}\left(E_{j}\right)=0$ for $i \neq j$. But then for $\left(x_{1}, \ldots, x_{n}\right) \in M$, $\left(x_{1}, \ldots, x_{n}\right)=e_{1}^{x_{1}}+\cdots+e_{n}^{x_{n}} \in B$, i.e., $M=B$.

Proof of III.4. Let $\psi$ be surjective and $\mathscr{U}=\{\{0\}\}$, but assume there is an $(M, M)$-subgroup $B$ of $M$ with $M_{c} \varsubsetneqq B \varsubsetneqq M$. Let $b=\left(b_{1}, \ldots, b_{n}\right)$ be a nonconstant function in $B$. Then $b^{\prime}=b-\left(b_{1}, \ldots, b_{1}\right)=\left(0, b_{2}-b_{1}, \ldots, b_{n}-\right.$ $\left.b_{1}\right)$ is in $B$. We show each $b_{j}-b_{1} \in \psi^{-1} \psi(0)$. Since $\psi$ is surjective, $\exists k_{i} \in K$ with $\psi\left(k_{i}\right)=E_{i}, i=1,2, \ldots, n$. Define $g \in M$ by $g\left(E_{1}\right)=k_{j}$ and $g\left(E_{i}\right)=k_{1}$ for $i \neq 1$. Then $b^{\prime \prime}=b^{\prime} * g \in B$ with $b^{\prime \prime}=\left(b_{j}-b_{1}, 0, \ldots, 0\right)$. Suppose $b_{j}-b_{1} \notin \psi^{-1} \psi(0)$, say $\psi\left(b_{j}-b_{1}\right)=E_{l} \neq \psi(0)$. Then, for $x \in K$, $e_{l}^{x} * b^{\prime \prime} \in B$ and $e_{l}^{x} * b^{\prime \prime}=(x, 0, \ldots, 0)$. This implies $A_{1}=\operatorname{Ann}\left(\bigcup_{j \neq 1} E_{j}\right) \subseteq B$, so by the above lemma, $B=M$. But this contradicts $B \varsubsetneqq M$ so we have $b_{j}-b_{1} \in \psi^{-1} \psi(0)$ for $j=1,2, \ldots, n$.

For $b \in B, b=\left(b_{1}, \ldots, b_{n}\right)$ we say $b_{i}$ belongs to $b$. Define $H=\left\{b_{j}-\right.$ $b_{i} \mid b_{j}, b_{i}$ belong to some $\left.b \in B\right\}$. Note first if $b_{j}-b_{i} \in H$ then $-\left(b_{j}-b_{i}\right)$ $=b_{i}-b_{j}$ is also in $H$. Now let $b_{j}-b_{i}, c_{l}-c_{m}$ be in $H$. From above, $\left(b_{j}-b_{i}, 0, \ldots, 0\right)$ and $\left(c_{l}-c_{m}, 0, \ldots, 0\right)$ are in $B$ which means $\left(b_{j}-b_{i}+\right.$ $\left.\left(c_{l}-c_{m}\right), 0, \ldots, 0\right)$ is in $B$, so $b_{j}-b_{i}+c_{l}-c_{m} \in H$. Hence $H$ is closed under addition, which means $H<K$. To show $H \unlhd K$, let $b_{j}-b_{i} \in H$. Thus for some $b \in B, b=\left(b_{1}, \ldots, b_{i}, \ldots, b_{j}, \ldots, b_{n}\right)$, so $c_{k}+b=\left(k+b_{1}, \ldots, k+\right.$ $\left.b_{i}, \ldots, k+b_{j}, \ldots, k+b_{n}\right) \in B$. Hence $k+b_{j}-\left(k+b_{i}\right) \in H$, which gives the result.

Now let $y \in K$ and $b_{j}-b_{i} \in H$. Then $\left(b_{j}-b_{i}, 0, \ldots, 0\right) \in B$ as does $\left(b_{j}-b_{i}+y, y, \ldots, y\right)$. Suppose $b_{j}-b_{i}+y \notin \psi^{-1} \psi(y)$, say $\psi\left(b_{j}-b_{i}+y\right)=$ $E_{l} \neq \psi(y)$. Then, as above, we get $A_{1} \subseteq B$ and, by III.5, $B=M$, again a contradiction to $B \varsubsetneqq M$. Therefore we must have $H+y \subseteq \psi^{-1} \psi(y)$ for each $y \in K$. We have found that $H \in \mathscr{U}$ and, since $B \supsetneqq M_{c}$, that $H \neq\{0\}$, which is a contradiction to $\mathscr{U}=\{\{0\}\}$. This means that $M$ must be $(M, M)$-simple. 
It remains to complete the characterization for the situation in which $|X / E|=$ 2. Thus we take $|X| E \mid=2$ and $\psi$ surjective. In the above proof we showed that $\mathscr{U}=\{\{0\}\}$ implies $M$ is $(M, M)$-simple. Furthermore, the discussion prior to III.4 points out that if $M$ is not $(M, M)$-simple then there exists $\{0\} \neq H \in \mathscr{U}$. If $H=K$ then $|\psi(K)|=1$, which is impossible since $\psi$ is surjective and $|X / E|=2$.

Corollary III.6. Let $|X / E|=2$ and $|\psi(K)|=2$. Then $M$ is $(M, M)$-simple $\Leftrightarrow \mathscr{U}=\{\{0\}\}$.

\section{ACTION OF $M$ on $K$}

For any group $G$ and any subnear-ring $N$ of the near-ring $M(G)$ of mappings on $G$, there is a natural action $N \times G \rightarrow G$ of $N$ on $G$ so that $G$ is an $N$-group. One then investigates various properties of this action. In this section we show that there exists a "natural" action of $M:=M(X / E, K, \psi)$ on $K$ and we initiate a study of the $M$-group $K$. Specifically, we characterize when $K$ is $\nu$-primitive, $\nu=0,1,2$.

We first give our action of $M$ on $K$. Let $f \in M, k \in K$ and define $M \times K \rightarrow K$ by $(f, k) \mapsto f(\psi(k))$, which we denote by $f \cdot k$. For $f, g \in M$ and $k \in K,(f+g) \cdot k=f \cdot k+g \cdot k$ while $(f * g) \cdot k=(f * g)(\psi(k))=$ $(f \psi g)(\psi(k))=f(\psi(g(\psi(k))))=f \cdot g(\psi(k))=f \cdot(g \cdot k)$. Hence $K$ is an $M$-group.

Suppose $f \cdot K=\{0\}$ for some $f \in M$. If $\psi$ is surjective then, since $f(\psi(k))=0$ for each $k \in K, f$ is the zero map in $M$. This shows that if $\psi$ is surjective then the action of $M$ on $K$ is faithful. If $\psi$ is not surjective, one can find $g \in M$ with $g(\psi(K))=\{0\}$ and $g(X / E-\psi(K)) \neq\{0\}$. Therefore, $g \cdot K=\{0\}$ and $K$ is not a faithful $M$-group.

Lemma IV.1. For $M=M(X / E, K, \psi), K$ is a faithful $M$-group $\Leftrightarrow \psi$ is surjective.

Lemma IV.2. For each $k \in K, M \cdot k=K$.

Proof. For each $a \in K$ the constant function $c_{a}$ is in $M$ and $c_{a} \cdot k=a$. Hence $K \subseteq M \cdot k$.

From this lemma we note that $K$ is a strongly monogenic $N$-group, consequently, $K$ is type 0 if and only if $K$ is a type 1 [5, p. 77].

Lemma IV.3. For each $k \in K, M_{0} \cdot k=\{0\}$ if $k \in \psi^{-1} \psi(0)$ and $K$ otherwise. Proof. We first note that $g \in M_{0} \Leftrightarrow g * 0=0 \Leftrightarrow g \psi(0)=0$. Now $M_{0} \cdot k=$ $\left\{g(\psi(k)) \mid g \in M_{0}\right\}$. If $k \in \psi^{-1} \psi(0)$ then $\psi(k)=\psi(0)$, so $g(\psi(k))=0$, i.e., $M_{0} \cdot k=\{0\}$. If $\psi(k) \neq \psi(0)$ then for any $k^{\prime} \in K$ there exists $g \in M_{0}$ with $g(\psi(k))=k^{\prime}$, hence $M_{0} \cdot k=K$.

If $K$ is a 2-primitive $M$-group then $K$ is a faithful $M$-group and of type 2 where "type 2 " means that $K$ has no nontrivial $M_{0}$-subgroups. Thus if $K$ is not of type 2 then there exists a subgroup $A$ of $K,\{0\} \neq A \neq K$ with $M_{0} \cdot A \subseteq A$. From the above lemma this means $A \subseteq \psi^{-1} \psi(0)$. The converse is also true. For if $C$ is a subgroup of $K\{0\} \neq C \neq \bar{K}$, such that $C \subseteq \psi^{-1} \psi(0)$, then again from the above lemma, $M_{0} \cdot C \subseteq C$, so $K$ is not of type 2 . 
Lemma IV.4. The group $K$ is an $M$-group of type $2 \Leftrightarrow$ there are no nonzero subgroups of $K$ contained in $\psi^{-1} \psi(0)$.

We say $\psi^{-1} \psi(0)$ is subgroup free if the conditions of the above lemma hold. Combining Lemmas IV.1 and IV.4 we obtain

Theorem IV.5. For $M=M(X / E, K, \psi), K$ is a 2-primitive $M$-group $\Leftrightarrow \psi$ is surjective and $\psi^{-1} \psi(0)$ is subgroup free.

We turn next to 1-primitivity and 0-primitivity. We recall that for any nearring $N$, an $N$-group $G$ is 0 -primitive if $G$ is a faithful $N$-group and $G$ is of type 0 , i.e., $G$ is a simple $N$-group. From the remark following Lemma IV.2, in our case, type 0 is equivalent to type 1 so we have that $K$ is 1 -primitive if and only if $K$ is 0 -primitive.

Suppose $K$ is not a simple $M$-group and let $A$ be an ideal of ${ }_{M} K,\{0\} \neq$ $A \neq K$. By definition, $A \unlhd K$. Moreover, for each $f \in M, k \in K$, and $a \in A, f \cdot(a+k)-f \cdot k \in A$. Assume for some $k^{\prime} \in K$ and $a^{\prime} \in A$ that $\psi\left(a^{\prime}+k^{\prime}\right) \neq \psi\left(k^{\prime}\right)$. Then, for $x \in K$, define $f_{x}: X / E \rightarrow K$ by $f_{x}\left(\psi\left(k^{\prime}\right)\right)=0$ and $f_{x} E_{\alpha}=x, E_{\alpha} \neq \psi\left(k^{\prime}\right)$. Then $f_{x} \cdot\left(a^{\prime}+k^{\prime}\right)-f_{x} \cdot k^{\prime}=f_{x}\left(\psi\left(a^{\prime}+k^{\prime}\right)\right)-$ $f_{x}\left(\psi\left(k^{\prime}\right)\right)=x$, a contradiction to $A \neq K$. This means that $\psi(a+k)=\psi(k)$, for each $k \in K, a \in A$, hence $A \in \mathscr{U}$.

Now let $K$ be 0 -primitive. Since $K$ is a faithful $M$-group, $\psi$ is surjective. Assume $\mathscr{U} \neq\{\{0\}\}$, say $\{0\} \neq B \in \mathscr{U}$. Since $B \in \mathscr{U}$, it follows that $B \unlhd K$. Let $f \in M, b \in B, k \in K$ and consider $f \cdot(b+k)-f \cdot k=$ $f(\psi(b+k))-f(\psi(k))$. Again, since $B \in \mathscr{U}$, it follows that $\psi(b+k)=\psi(k)$, so $f \cdot(b+k)-f \cdot k=0 \in B$. Thus $B$ is a nonzero ideal of ${ }_{M} K$ and, since $K$ is 0 -primitive, $B=K$. But, as we have seen, when $\psi$ is surjective, $K \notin \mathscr{U}$. We conclude that $\mathscr{U}=\{\{0\}\}$. We have established the following result.

Theorem IV.6. Let $M=M(X / E, K, \psi)$. The following are equivalent:

(i) ${ }_{M} K$ is 0-primitive;

(ii) ${ }_{M} K$ is 1-primitive;

(iii) $\psi$ is surjective and $\mathscr{U}=\{\{0\}\}$.

We combine Theorems III.2, III.4, and IV.6 to obtain our main characterization result.

Corollary IV.7. Let $M=M(X / E, K, \psi)$ with $3 \leq|X / E|<\infty$. The following are equivalent:

(i) ${ }_{M} K$ is 0-primitive;

(ii) ${ }_{M} K$ is 1-primitive;

(iii) $M$ is a simple near-ring,

(iv) $M$ is $(M, M)$-simple;

(v) $\psi$ is surjective and $\mathscr{U}=\{\{0\}\}$.

In the proof of Theorem IV.6 we showed that if $A$ is an ideal of ${ }_{M} K$ then $A \in \mathscr{U}$ or $A=K$ and conversely, if $B \in \mathscr{U} \cup\{K\}$ then $B$ is an ideal of ${ }_{M} K$. This gives a determination of the ideals of ${ }_{M} K$.

Corollary IV.8. If $\mathscr{J}\left({ }_{M} K\right)$ denotes the lattice of $M$-ideals of ${ }_{M} K$, then there exists a lattice isomorphism between $\mathscr{J}\left({ }_{M} K\right)$ and $\mathscr{U} \cup\{K\}$. 


\section{REFERENCES}

1. P. Fuchs, On the structure of ideals in sandwich near-rings, Resultate Math. 17 (1990), 256-271.

2. P. Fuchs and G. Pilz, A new density theorem for primitive near-rings (submitted).

3. C. J. Maxson and L. van Wyk, Near-rings of invariants, Resultate Math. 18 (1990), 286-297.

4. J. D. P. Meldrum, Near-rings and their links with groups, Research Notes in Math., vol. 134, Pitman, London, 1986.

5. G. Pilz, Near-rings, 2nd ed., North-Holland, Amsterdam, 1983.

6. H. Wielandt, Permutation groups through invariant relations and invariant functions, Lecture Notes, Ohio State Univ., 1969.

Department of Mathematics, Texas A \& M University, College Station, Texas 77843 